\title{
Análisis comparativo del área y morfología foliar en taxones ibéricos amenazados del género Coincya (Cruciferae)
}

\author{
Comparative analysis of leaf area and morphology in threatened Iberian Coincya \\ (Cruciferae) taxa
}

\author{
MIGUEL A. COPETE ${ }^{1, ~ *}$, JOSE M. HERRANZ1 \& PABLO FERRANDIS ${ }^{1}$ \\ ${ }^{1}$ Departamento de Producción Vegetal y Tecnología Agraria, E.T.S., Ingenieros Agrónomos, Universidad de Castilla-La \\ Mancha, Campus Universitario s/n, 02071, Albacete, España. \\ * Autor correspondiente: miguel.copete@uclm.es
}

\begin{abstract}
RESUMEN
Se evalúa la variación del parámetro área foliar y un índice de morfología foliar (índice de forma) en plantas de 1,2 y 3 meses de edad pertenecientes a cuatro taxones del género Coincya (Cruciferae) relacionados filogenéticamente. Para todas las edades el área foliar alcanzó sus valores más altos en las formas que tienen frutos con rostros gruesos (C. rupestris), aumentando progresivamente según el siguiente orden: $C$. monensis subsp. orophila, $C$. longirostra, C. rupestris subsp. leptocarpa y C. rupestris subsp. rupestris. Se plantea que el aumento del área foliar en los taxones cuyos frutos poseen rostros gruesos puede constituir otra ventaja adaptativa adicional a las ya mencionadas para estos en trabajos anteriores a la vez que refuerza el esquema evolutivo propuesto para la totalidad del grupo. En cuanto a la morfología foliar, el índice de forma discrimina nítidamente los taxones extremos de esta línea filogenética: $C$. rupestris subsp. rupestris (más evolucionado) y C. monensis subsp. orophila (más arcaico) una vez alcanzada la fase adulta; las formas intermedias, representadas por $C$. rupestris subsp. leptocarpa y $C$. longirostra, quedan encuadradas en un mismo grupo casi en su totalidad.
\end{abstract}

Palabras clave: área foliar, morfología foliar, rostro engrosado, ventaja adaptativa.

\begin{abstract}
Variation in leaf area and in a leaf morphological index (shape index) was evaluated in seedlings 1, 2, and 3-month aged in four taxa of Coincya genus (Cruciferae) phylogenetically related. For all ages, leaf area reached higher values in types having thick-beak fruits (C. rupestris), and gradually increased according to the following order: $C$. monensis subsp. orophila, C. longirostra, C. rupestris subsp. leptocarpa, and C. rupestris subsp. rupestris. We suggest that the increase of leaf area in thick-beak taxa may be an adaptive advantage, additional to those already suggested in previous studies, reinforcing as well the evolutive schema proposed for the entire group. With regard to leaf morphology, shape index neatly discriminated extreme taxa in the phylogenetical line (i.e., C. rupestris subsp. rupestris in the most evolved point versus $C$. monensis subsp. orophila as the most archaic taxon) when plants reach the adult phase. Intermediate types (i.e., C. rupestris subsp. leptocarpa and C. longirostra) are included within the same group nearly overall.
\end{abstract}

Key words: adaptive advantage, leaf area, leaf morphology, thick fruit-beak.

\section{INTRODUCCIÓN}

Cuatro taxones del género Coincya (Cruciferae, tribu Brassiceae) habitan en el área central del sur de la Península Ibérica a lo largo de la alineación montañosa de Sierra Morena y la vecina Sierra de Alcaraz: Coincya rupestris Porta \& Rigo subsp. rupestris, $C$. rupestris Porta \& Rigo subsp. leptocarpa (Glez. Albo) Leadlay, C. longirostra (Boiss.) Greuter \& Burdet y $C$. monensis (L.) Greuter \& Burdet subsp. orophila (Franco) Aedo, Leadlay \& Muñoz Garm. Los tres primeros, considerados amenazados, están incluidos en la más reciente Lista Roja de la Flora Vascular Española (Moreno 2008), con las siguientes categorías UICN (UICN 2001): "En peligro crítico" para C. rupestris subsp. rupestris, "En peligro" para C. longirostra, y "Vulnerable" para $C$. rupestris subsp. leptocarpa. Además, $C$. rupestris subsp. rupestris está también incluida en el Anexo II de la Directiva CEE 43/1992, "Directiva Hábitat" (DOCE 1992) y, asimismo, tiene plan de recuperación aprobado por el gobierno autónomo de Castilla-La Mancha (DOCM 1999). 
En un primer trabajo sobre características morfológicas del fruto en estos taxones, Gómez-Campo (1977) mostró la existencia de una variación clinal entre poblaciones occidentales con silicuas con una porción indehiscente (rostro) corta y delgada $(C$. monensis subsp. orophila) y poblaciones orientales con rostros mayores que la porción dehiscente del fruto (valvas) y más gruesos que estas (C. rupestris subsp. rupestris).

Posteriormente, Gómez-Campo et al. (2001) realizaron un análisis morfométrico partiendo de 11 parámetros medidos en frutos maduros y establecieron un esquema de las posibles relaciones evolutivas dentro del grupo según el cual de las formas más antiguas, representadas por $C$. monensis subsp. orophila, se llegaría a las más modernas, representadas por $C$. rupestris subsp. rupestris, a través de $C$. longirostra primero y de $C$. rupestris subsp. leptocarpa en una fase posterior de evolución. Asimismo, estos autores indicaron que en los taxones con rostros gruesos (C. rupestris subsp. rupestris y C. rupestris subsp. leptocarpa) el ratio " $\mathrm{n}^{\circ}$ semillas del rostro / $\mathrm{n}^{\circ}$ de semillas de las valvas" es mayor que en los taxones con rostros delgados, a la vez que señalaron un conjunto de ventajas adaptativas de las formas con rostros gruesos en relación a las formas con rostros delgados: a) facilitación de la dispersión en el tiempo, provocando una germinación diferida de las semillas del rostro con relación a las de las valvas, ya que es preciso el transcurso de un cierto tiempo hasta que las semillas consiguen liberarse de las paredes del rostro indehiscente, b) facilitación de dispersión de los rostros en el espacio, bien por medio del viento o de las aguas de escorrentía, lo que puede permitir depositar las semillas que contengan en grietas de rocas alejadas de la planta madre que las ha originado, c) las semillas embutidas en el interior de rostros gruesos pueden resistir las altas temperaturas $\left(120^{\circ} \mathrm{C}\right)$ producidas por los incendios forestales, fenómeno frecuente en la región mediterránea. Por otra parte, Herranz et al. (2003) indicaron que en las formas de rostros gruesos las semillas son mayores que en las formas de rostros delgados, así como que, en aquellas, las semillas contenidas en los rostros son 1.7-2.3 veces más pesadas que las contenidas en las valvas. Este mayor contenido en reservas de las semillas de las formas con rostros gruesos puede traducirse en un incremento de la probabilidad de supervivencia de plántulas, como ha sido probado para otras especies de crucíferas (Krannitz et al. 1991, Maun \& Payne 1998). Esta característica de las formas con rostros gruesos sería otra ventaja adaptativa a añadir a las indicadas por GómezCampo et al. (2001), sin embargo, en dichas formas, la mayor capacidad germinativa de las semillas contenidas en rostros en relación a la de las valvas solo ha podido ser verificada en dos de las tres poblaciones analizadas (Copete et al. 2005).

La importancia del área foliar como ventaja adaptativa ha sido puesta de manifiesto en especies pertenecientes a diferentes familias. Lüscher et al. (2001) comprobaron que en Trifolium repens la capacidad de competencia y supervivencia al periodo frío aumentaban con el área foliar, y Bossdorf et al. (2004) verificaron el descenso en el éxito reproductivo de Alliaria petiolata con la reducción del área foliar. Por ello, la principal finalidad de este trabajo es comprobar si los taxones de Coincya con frutos de rostros gruesos, y por tanto más evolucionados, tienen mayor área foliar, a igualdad de edad, que los taxones con frutos de rostros delgados. Si esta hipótesis se verificara sería una ventaja adaptativa adicional a las ya mencionadas. Por otro lado, se trata de analizar la morfología foliar como un posible carácter con valor taxonómico, que permita determinar ejemplares de estos taxones sin necesidad de recurrir al fruto. Ello resultaría interesante en estos taxones bienales que fructifican durante su segundo año de vida y que, por tanto, pasan la mayor parte de la misma en fase de roseta vegetativa, dificultándose su determinación. Simultáneamente se pretende observar la jerarquía de variación entre los diferentes taxones en función del parámetro morfológico diseñado para el objetivo anterior, que de ser similar a la obtenida en relación al área foliar, podría interpretarse como un refuerzo de la posible tendencia evolutiva sugerida. Paralelamente al análisis de área y morfología foliar, y aprovechando la disponibilidad de plantas de los cuatro taxones cultivadas bajo las mismas condiciones ambientales, se ha considerado conveniente evaluar también el tiempo necesario para que se produzca la floración. Por lo tanto, los objetivos de este trabajo son: 
a) Analizar la variación del parámetro área foliar con la edad de las plántulas en los cuatro taxones de Coincya considerados en este trabajo; b) en las mismas plantas consideradas en el objetivo anterior, calcular un parámetro descriptor del contorno foliar que nos permita tanto su empleo con carácter taxonómico, como jerarquizar los cuatro taxones considerados basándonos en el mismo; c) evaluar el tiempo preciso para el inicio de la floración.

\section{MÉTODOS}

\section{Material vegetal}

En la segunda quincena de junio de 2000 se procedió a la recogida de semillas de los diferentes taxones en las siguientes localidades de la Península Ibérica (Fig. 1):
C. rupestris subsp. rupestris (La Molata, MO, UTM: 30S-WH4677, $1040 \mathrm{~m}$, en Alcaraz provincia de Albacete), C. rupestris subsp. leptocarpa (Sierra de Relumbrar, RL, UTM: 30S-WH2771, $860 \mathrm{~m}$, en Villapalacios provincia de Albacete), C. longirostra (Despeñaperros, DS, UTM: 30S-VH5651, $720 \mathrm{~m}$, en Viso del Marqués provincia de Ciudad Real) y $C$. monensis subsp. orophila (Puerto de Niefla, NF, UTM: 30S-UH7966, $880 \mathrm{~m}$, en Brazatortas provincia de Ciudad Real). Para cada población se indica su clave correspondiente con la que figura en los trabajos previos publicados (Gómez-Campo et al. 2001, Herranz et al. 2003, Copete et al. 2005). La nomenclatura sigue a Castroviejo et al. 1986-2003. Tras dejar extendidas durante unos días las semillas en laboratorio a temperatura ambiente $\left(20^{\circ} \mathrm{C}\right)$, se seleccionaron las que ofrecían aspecto sano y estaban libres de parásitos, y se guardaron en cámara frigorífica a $5{ }^{\circ} \mathrm{C}$ hasta que fueron requeridas para la siembra.

\section{Diseño experimental y adquisición de datos}

La siembra se llevó a cabo el 20 de septiembre de 2000 en macetas de $21 \mathrm{~cm}$ de diámetro y $25 \mathrm{~cm}$ de

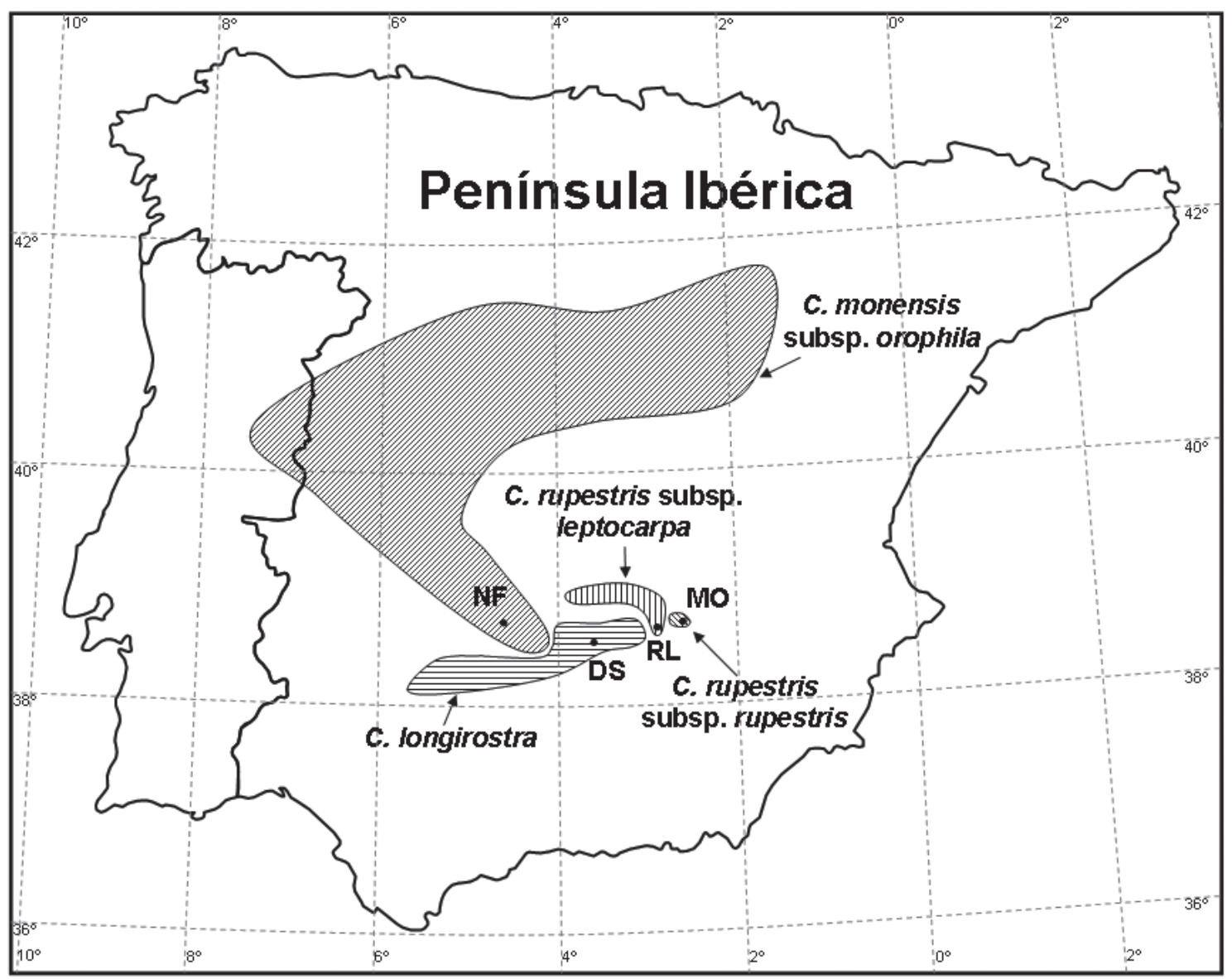

Fig. 1: Áreas de distribución aproximadas en la Península Ibérica de los cuatro taxones de Coincya estudiados. En cada una de ellas se indica con un punto la ubicación de la localidad de procedencia del material vegetal empleado.

Approximate distribution areas of the Coincya taxa studied in the Iberian peninsula. The position of a point shows the origin of the plant material in each area. 
profundidad $\left(6 \mathrm{dm}^{3}\right)$, con un sustrato constituido por una mezcla de turba enriquecida en nutrientes y arena en proporción $2: 1$, quedando las macetas ubicadas en un umbráculo a temperatura ambiente. Se sembraron 60 macetas por taxón, con 4-6 semillas por maceta, seleccionando en cada maceta la plántula más vigorosa y centrada de entre las emergidas, produciéndose la nascencia en la primera semana de octubre de 2000.

Para cada taxón se evaluó la superficie foliar y el número de hojas para las edades de uno, dos y tres meses, contadas estas a partir de la emergencia de las plántulas. Ensayos preliminares realizados en 1999 mostraron que aparentemente a partir de los tres meses de edad las diferentes hojas forman una roseta basal cuyo crecimiento vegetativo se ralentiza notablemente. Para medir el área foliar se utilizó un método destructivo consistente en arrancar la planta, separar todas las hojas integrantes de cada roseta basal a la altura de la inserción del peciolo con el tallo, para proceder después al prensado cuidadoso de cada hoja hasta su secado completo (8-10 días). De las 50 plantas iniciales por taxón, como consecuencia de la muerte de alguna de ellas, el número de ejemplares analizado para cada una de las edades fue el siguiente: 25 plantas con un mes de edad por taxón, entre seis y 15 plantas con dos meses (seis plantas de C. rupestris subsp. rupestris y $C$. monensis subsp. orophila, $10 \mathrm{de} C$. rupestris subsp. leptocarpa y 15 de $C$. longirostra), y finalmente seis plantas con tres meses de edad por taxón. La disminución del tamaño muestral con la edad fue debida al incremento exponencial de trabajo que supone la medición de las áreas foliares con la edad de las plantas, llegando a ser una labor considerablemente tediosa en plantas de tres meses con muchas hojas y estas de grandes dimensiones. Asimismo, de cada taxón se reservaron 10 plantas vivas vigorosas con objeto de ratificar la no prolongación del crecimiento vegetativo más allá de los tres meses de edad, para lo cual se registró el diámetro de las rosetas basales y el número de hojas a la edad de tres meses y posteriormente a los cuatro y cinco meses. Esta muestra de plantas vivas también nos sirvió para registrar el inicio de la floración en cada uno de los cuatro taxones.

De la totalidad de las hojas prensadas correspondientes a cada planta se midieron los parámetros área y perímetro mediante el programa GLOBAL LAB IMAGE/2 versión 3.0 (Data Translation 2001), previa digitalización de las imágenes de las hojas mediante escáner (HP 5300C, resolución 1200 dpi). Esta metodología facilita las mediciones, a la vez que evita los errores en el cálculo del área foliar detectados por Tsuda (1999) para otras técnicas.

La morfología foliar se cuantificó mediante el índice de forma, definido como el ratio entre el perímetro de la hoja y la raíz cuadrada de su área (Kincaid \& Schneider 1983; McLellan 1993, 2000, 2005, McLellan \& Endler 1998). Este índice resultó ser el más eficiente entre otros muchos testados por ser el que presenta una relación más elevada entre simplicidad de cálculo/precisión en las estimaciones (McLellan \& Endler 1998). El valor correspondiente a una planta se obtiene a partir de su área foliar total y de la suma de los perímetros de todas las hojas que la integran. Definido de esta manera, cuanto mayor es este factor más lobulado es el limbo de las hojas, por lo que puede utilizarse para detectar diferencias morfológicas entre los distintos taxones, así como la escala de variación entre los mismos. En los cuatro taxones del género Coincya evaluados las diferencias en lobulación de las hojas están más marcadas en ejemplares adultos e individuos juveniles (Fig. 2) que en el caso de plántulas (Fig. 3).

El utilizar material vegetal procedente de plantas cultivadas y no de plantas recolectadas en su hábitat natural, tiene como finalidad el permitir un control más exhaustivo de la edad de las plantas e impedir daños por herbivoría. Por otra parte, tanto el área como la forma foliar son caracteres hereditarios en una proporción elevada, y por tanto indicadores fiables del genotipo

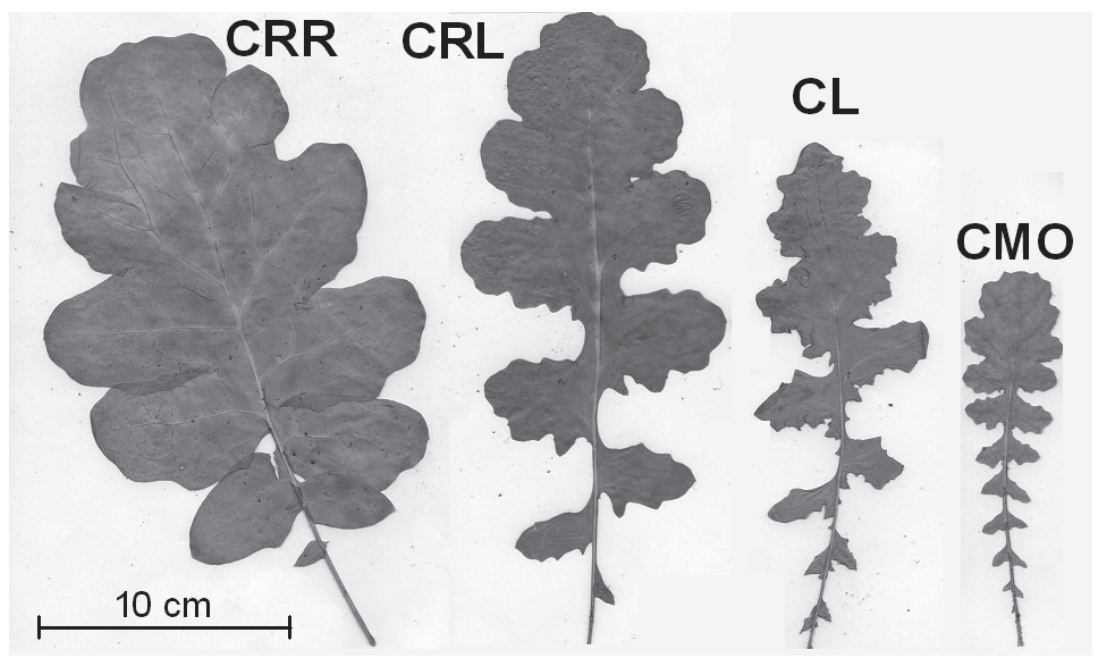

Fig. 2: Hojas individuales correspondientes a plantas con tres meses de edad de los taxones de Coincya estudiados: CRR (C. rupestris subsp. rupestris), CRL (C. rupestris subsp. leptocarpa), CL (C. longirostra) y CMO (C. monensis subsp. orophila).

Single leaves of three-month-old plants in the Coincya taxa studied: CRR (C. rupestris subsp. rupestris), CRL (C. rupestris subsp. leptocarpa), CL (C. longirostra) and CMO (C. monensis subsp. orophila). 
independientemente de las condiciones ambientales en las que se desarrolla la planta (McLellan 2000).

Para evaluar el tiempo transcurrido hasta la floración se ha tenido en cuenta que esta se inicia a finales de invierno-principios de primavera, y que en la mayoría de los taxones analizados no se produce hasta el segundo ciclo vegetativo. C. monensis subsp. orophila es el único taxón del grupo que puede comportarse a veces como anual de invierno (Leadlay 1993). Dado que en el caso de este estudio las plantas supervivientes no iniciaron su floración hasta finales de invierno-principios de primavera de 2002, en cada planta individual se ha tomado como tiempo hasta la floración el número de días transcurridos desde el inicio del segundo invierno postemergencia (21diciembre-2001) hasta la antesis de la primera flor.

\section{Análisis estadístico}

La comparación de los valores de área foliar y número de hojas requirió las verificaciones previas de normalidad (test de Cochran) y homocedasticidad (test de David). Para mejorar su ajuste a una distribución normal, los valores de ambos parámetros fueron transformados mediante la función raíz cuadrada y posteriormente sometidos a un ANOVA factorial, con los factores taxón y edad. La prueba de Tukey (1949) fue usada para detectar diferencias significativas $(\alpha=$ $0.05)$.

En cuanto a la morfología foliar, y considerando cada edad por separado, se han agrupado los ejemplares analizados de los cuatro taxones en bloques homogéneos en función del índice de forma, de modo que los individuos encuadrados en un mismo grupo posean cierta similitud en cuanto a la forma de sus hojas, independientemente del taxón al que pertenezcan. Con tal finalidad se aplicó un análisis cluster mediante el método del centroide y en base a la distancia euclídea al cuadrado.

\section{RESULTADOS}

\section{Área foliar}

En relación al parámetro área foliar, se observa una progresiva diferenciación entre taxones conforme aumenta la edad de las plantas, de modo que con un mes de edad no existen diferencias, con dos meses se inicia la diferenciación al presentar $C$. rupestris subsp. rupestris mayor área foliar que $C$. longirostra y C. monensis subsp. orophila, siendo ya con tres meses las diferencias significativas entre los cuatro taxones según el siguiente orden: $C$. monensis subsp. orophila $<$. longirostra $<$. . rupestris subsp. leptocarpa $<$ C. rupestris subsp. rupestris (Fig. 4).

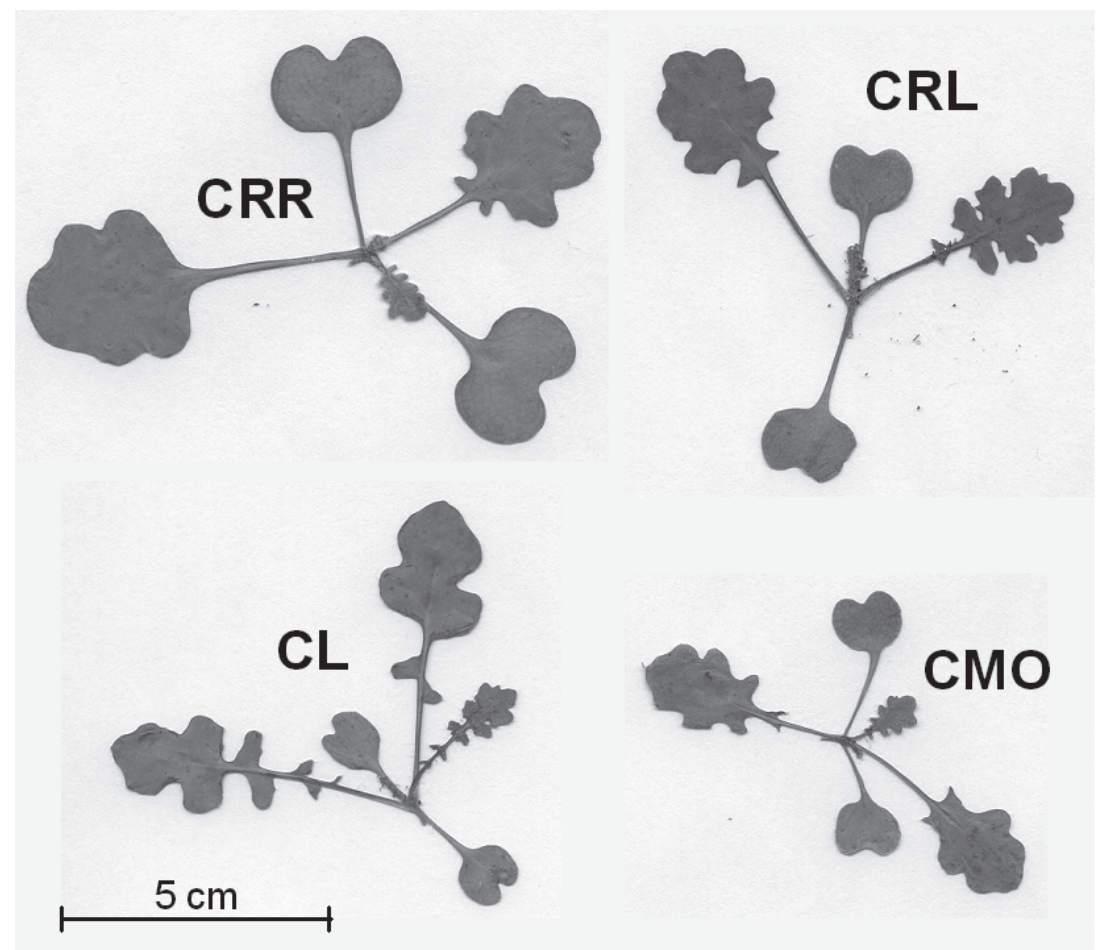

Fig. 3: Plántulas completas con un mes de edad de los taxones de Coincya estudiados: CRR (C. rupestris subsp. rupestris), CRL (C. rupestris subsp. leptocarpa), CL (C. longirostra) y CMO (C. monensis subsp. orophila).

Complete one-month-old seedlings of the Coincya taxa studied: CRR (C. rupestris subsp. rupestris), CRL (C. rupestris subsp. leptocarpa), CL (C. longirostra) and CMO (C. monensis subsp. orophila). 
Respecto al número medio de hojas por planta, en la Fig. 4 se observan diferencias menos acentuadas que en relación al área foliar, en ocasiones sin llegar a ser significativas. No obstante, en términos generales se verifica la tendencia por la que plantas de mayor área tienen más hojas. De las dos variables anteriores se deriva el tamaño de la hoja media, que para la edad de 3 meses alcanza los siguientes valores (media \pm error estándar) que se ajustan a la misma gradación de taxones referida en el párrafo anterior: 20.9 $\pm 1.5^{\mathrm{a}}, 41.8 \pm 3.5^{\mathrm{ab}}, 60.8 \pm 3.5^{\mathrm{b}}, 101.0 \pm 13.9^{\mathrm{c}}$ (todos los valores expresados en $\mathrm{cm}^{2}$; letras distintas denotan diferencia significativas, $\mathrm{F}_{3,23}$ $=21.23, \mathrm{P}<0.0001$ ).

\section{Morfología foliar}

En lo que se refiere al parámetro morfológico índice de forma, en los dendrogramas correspondientes al análisis cluster relativo a las plantas de uno y dos meses de edad no se aprecia una segregación clara de los individuos pertenecientes a cada taxón (datos no mostrados). En cambio, en plantas con tres meses de edad (Fig. 5) esta diferenciación es evidente en ejemplares de $C$. rupestris subsp. rupestris (gupos 1 y 3) y $C$. monensis subsp. orophila (grupo 5) que se separan del resto, pero no así entre ejemplares de $C$. rupestris subsp. leptocarpa y $C$. longirostra que se entremezclan entre sí (grupo 4).

Los valores medios ( \pm error estándar) del índice de forma correspondiente a cada taxón a la edad de tres meses son: $23.3 \pm 0.8$ ( $C$. rupestris subsp. rupestris), $35.8 \pm 2.0$ (C. rupestris subsp. leptocarpa), $38.3 \pm 2.9$ (C. longirostra) y $30.3 \pm 0.9$ (C. monensis subsp. orophila). Para la edad de dos meses el índice de forma de los cuatro taxones se situó en el rango 18.0-21.3 y con un mes de edad varió entre 9.1-12.1.

A partir del tercer mes de edad el crecimiento vegetativo se detiene, de modo que no se registraron ni incrementos en el número de hojas, ni en el tamaño de la roseta basal.

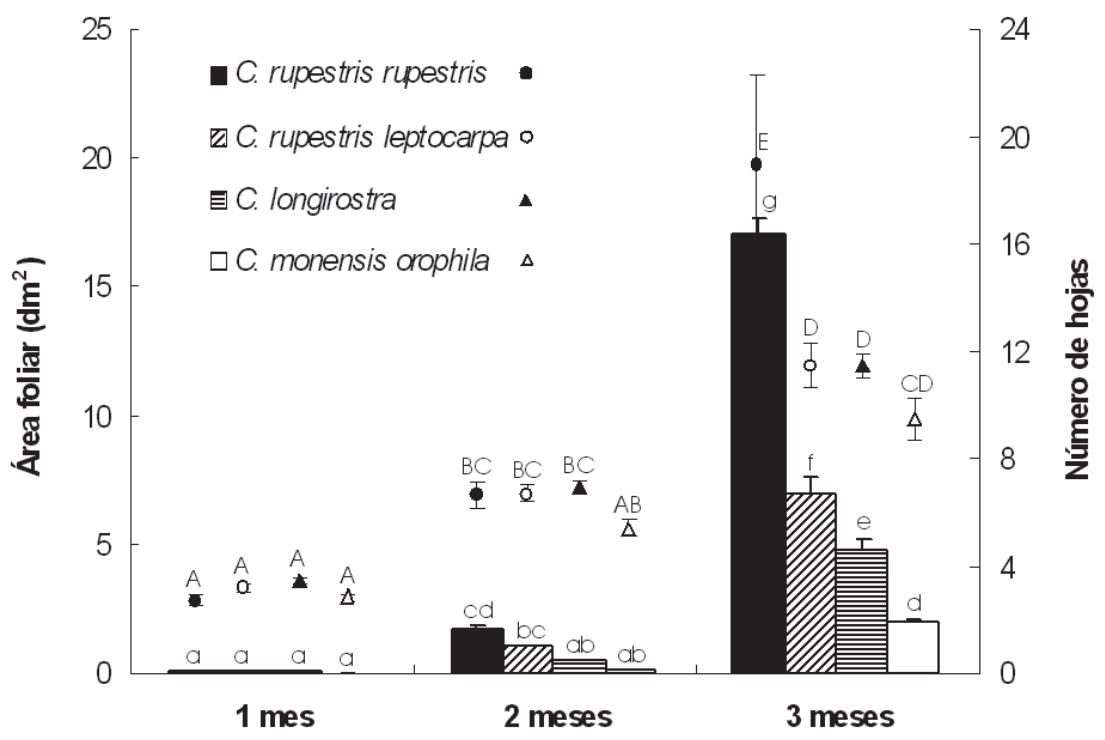

Fig. 4: Valores medios del área foliar (columnas) y número de hojas por planta (puntos) para cada taxón en individuos de uno, dos y tres meses. Las barras verticales representan el error estándar correspondiente a cada media. Letras minúsculas diferentes denotan diferencias significativas según el parámetro área foliar entre taxones y edades, y letras mayúsculas diferentes revelan diferencias significativas en el número de hojas entre taxones y edades.

Mean leaf area (columns) and number of leaves per plant (points) for the Coincya taxa studied at one, two, three-month-old plants. Bars on columns represent the standard error. Values of leaf area followed by the same lower case letter are not significantly different from each other. Values of number of leaves followed by the same upper case letter are not significantly different from each other. 


\section{Tiempo hasta la floración}

Tomando como referencia el inicio del segundo invierno postemergencia, el número medio de días transcurridos hasta el inicio de la floración en cada taxón ha sido el siguiente: $76.5 \pm 2.5^{\text {a }}$ (C. rupestris subsp. rupestris), $94.2 \pm$ $4.3^{\mathrm{b}}$ (C. rupestris subsp. leptocarpa), $103.0 \pm$ $3.5^{\mathrm{b}}$ (C. longirostra), $102.8 \pm 3.6^{\mathrm{b}}$ ( $C$. monensis subsp. orophila). Letras distintas denotan diferencias significativas $\left(\mathrm{F}_{3,11}=11.95, \mathrm{P}<\right.$ 0.0001).

\section{DISCUSIÓN}

La jerarquización de los cuatro taxones de Coincya estudiados en base a su morfología foliar difiere de la obtenida a partir del área foliar. Si comparamos la clasificación derivada de cada uno de los dos parámetros mencionados, con la serie evolutiva propuesta por Gómez-Campo et al. (2001), se deduce que es el área foliar el parámetro cuya secuencia de variación entre taxones se ajusta a dicha serie evolutiva: $C$. monensis subsp. orophila < C. longirostra $<$ C. rupestris subsp. leptocarpa $<$ C. rupestris subsp. rupestris. El taxon más evolucionado, C. rupestris subsp. rupestris, presenta la mayor área foliar mientras que, $C$. monensis subsp. orophila, el taxón considerado más arcaico, la menor. Así, plantas de $C$. rupestris subsp. rupestris con dos meses de edad presentan un área foliar similar a la de $C$. monensis subsp. orophila con un mes más (tres meses). Por lo tanto, la mayor superficie foliar podría ser una ventaja adaptativa más a añadir a las ya mencionadas para las formas de rostros gruesos, tal y como sucede en Trifolium repens (Lüscher et al. 2001), Alliaria petiolata (Bossdorf et al. 2004), y en varios géneros con hojas planas caducas (Walter 1977). Por otra parte, previamente se ha verificado el mismo orden de variación entre taxones del parámetro "peso de las semillas" (Herranz et al. 2003). Estos resultados coinciden con los obtenidos por Marañón \& Grubb (1993) con plantas anuales mediterráneas en las que a semillas más grandes corresponden áreas foliares mayores y hojas más gruesas. Según estos autores, las especies de semillas mayores pueden desarrollar niveles más elevados de fotosíntesis por unidad de luz interceptada gracias a estas hojas más gruesas, lo que refuerza la consideración de la mayor superficie foliar de C. rupestris subsp. rupestris como una ventaja evolutiva.

El adelanto en la floración de $C$. rupestris subsp. rupestris respecto al resto de taxones puede considerarse también como una ventaja adaptativa en ecosistemas mediterráneos al

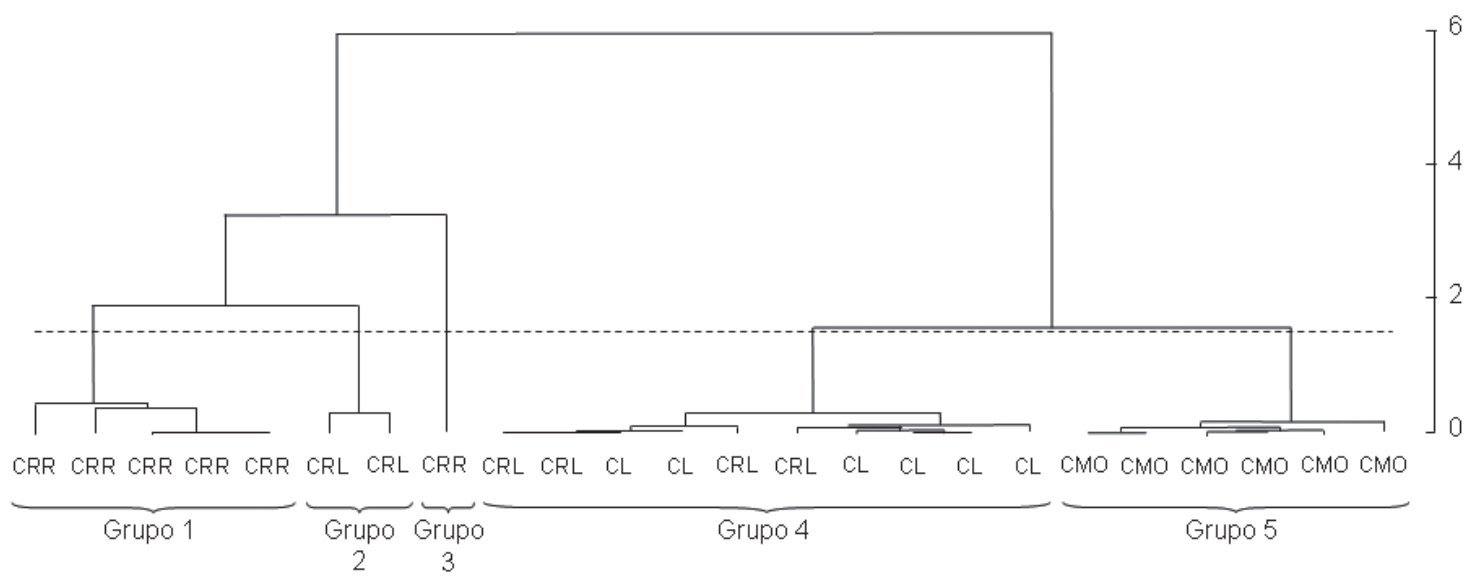

Fig. 5: Dendrograma resultante del análisis cluster de los resultados del índice de forma correspondiente a plantas con tres meses de edad de los taxones estudiados: CRR (C. rupestris subsp. rupestris), CRL $(C$. rupestris subsp. leptocarpa), CL (C. longirostra) y CMO (C. monensis subsp. orophila). Se indican las agrupaciones resultantes considerando una distancia de 1.5 (línea discontinua).

Dendrogram of the cluster analysis of the shape index values from 3-month-old plants of the Coincya taxa studied: CRR (C. rupestris subsp. rupestris), CRL (C. rupestris subsp. leptocarpa), CL (C. longirostra) and CMO (C. monensis subsp. orophila). Identified groups correspond to a distance level of 1.5 (dotted line). 
posibilitar la formación de las semillas antes de que el periodo de sequía característico de este clima lo impida. Estas diferencias respecto al inicio de la floración son de base genética, ya que la influencia medioambiental a través del proceso vernalización (Chouard 1960, Erwin 2005) no tendrá efecto al haberse desarrollado todas las plantas estudiadas bajo las mismas condiciones de cultivo. Pese a no haberse detectado diferencias significativas al respecto entre los taxones restantes, se observa una tendencia según la cual la floración de $C$. rupestris subsp. leptocarpa es más temprana que la de $C$. longirostra y $C$. monensis subsp. orophila, corroborando su mayor grado evolutivo. En el hábitat natural de C. rupestris subsp. rupestris también se han detectado variaciones interpoblacionales en lo que al inicio de la floración se refiere, confirmándose que la población con una floración más prematura presentó una mayor proporción de flores que originaron fruto (Copete et al. 2008).

Por el contrario, en relación al índice de forma analizado, que como ya se ha indicado muestra un orden de variación entre taxones diferente al del área foliar y otros parámetros tales como anchura del rostro y peso de las semillas, dicho índice alcanza su valor mínimo en $C$. rupestris subsp. rupestris, lo que equivale a decir que sus hojas son las menos lobuladas del grupo. Según algunos autores (Hickey \& Wolfe 1975, Baker-Brosh \& Peet 1997) las hojas lobuladas suponen un avance evolutivo por constituir una adaptación a temperaturas extremas y precipitaciones estacionales, y por tanto, bajo este punto de vista no tendría validez el esquema evolutivo propuesto. No obstante, los resultados globales obtenidos vienen a reforzar el esquema evolutivo sugerido por Gómez-Campo et al. (2001), dado que la morfología foliar se considera un parámetro de menor trascendencia ecológica que el área foliar (Givnish 1987), a la vez que también contribuyen a ello los valores obtenidos de tiempo de floración.

Para los cuatro taxones del grupo se verifica que con la edad de la planta la superficie foliar se incrementa de forma exponencial, y el índice de forma aumenta indicándonos una mayor lobulación de la hoja en plantas adultas. Asimismo, el tamaño medio de la hoja, para los cuatro taxones se incrementa con la edad, a diferencia de las observaciones realizadas por algunos autores indicando que las plantas de mayor edad con frecuencia poseen hojas más pequeñas que las jóvenes (Givnish 1987). La velocidad de crecimiento del área foliar fue máxima entre los 2-3 meses de edad. Resultados similares fueron obtenidos por Nanda et al. (1995) con varias especies del género Brassica, perteneciente a la misma tribu que Coincya, cuando fueron cultivadas en condiciones óptimas en siembras efectuadas a principios de otoño al igual que en el presente trabajo, a diferencia de siembras de especies de este mismo género, que realizadas a final de primavera presentaron un crecimiento máximo entre los 1-2 meses (Kasa \& Kondra 1986). Transcurridos los tres primeros meses desde la emergencia, apenas creció el diámetro de la roseta basal que forman las hojas de cada planta, lo que indica que bajo condiciones de cultivo alcanzan a los tres meses de edad un grado similar de desarrollo al logrado en la naturaleza a los nueve meses (Herranz et al. 1996), probablemente como consecuencia de la escasez de recursos en los hábitats rupícolas típicos de estos taxones (Lavergne et al. 2003).

Aunque los resultados obtenidos en cuanto a morfología foliar permiten separar fácilmente a los taxones más extremos del grupo ( $C$. rupestris subsp. rupestris y $C$. monensis subsp. orophila), la discriminación de $C$. longirostra y C. rupestris subsp. leptocarpa no resulta tan obvia, lo que desaconseja la utilización del valor de dicho parámetro como criterio taxonómico. No obstante, si tenemos en cuenta que se han encontrado correlaciones entre rasgos estructurales de las hojas y condiciones ambientales (Givnish 1987, Wilf 1997), podría ser interesante desde el punto de vista taxonómico el realizar estudios similares a este pero a partir de ejemplares desarrollados en su hábitat natural típico, para ver si las condiciones ambientales propias de cada hábitat confieren a los taxones de Coincya, en ellos desarrollados, ciertos rasgos morfológicos a nivel de hoja susceptibles de ser discriminados con el factor de forma aquí aplicado.

Si bien $C$. rupestris subsp. rupestris pudiera representar la forma más evolucionada del grupo y con mayores ventajas adaptativas es, sin embargo, el taxón con distribución 
geográfica más restringida y mayor grado de amenaza. Como sucede con la especie amenazada Banksia goodii (Witkowski \& Layout 1997) su rareza pudiera ser el resultado de su origen reciente, su hábitat característico sobre grietas y repisas de roquedos, y la fragmentación del mismo por la actividad humana que han impedido la colonización de su área potencial. Asimismo, un origen reciente, sin tiempo suficiente para expandirse, ha sido señalado como posible causa de la rareza de las especies de distribución restringida Echinacea tennesseensis (Baskin et al. 1997) y Solidago shortii (Walck et al. 2001). La baja capacidad competitiva no debe de ser una de las principales causas de rareza de $C$. rupestris subsp. rupestris, como ocurre con otras especies amenazadas de hábitats rupícolas (Baskin \& Baskin 1988, Walck et al. 1999, Lavergne et al. 2003), dado que posee mayor talla, área foliar, capacidad de producción de flores y frutos, y semillas más grandes que sus congéneres con distribución geográfica más amplia (Copete 2005).

Dado el peligro de desaparición que corren varias de las poblaciones de estos taxones, es importante la puesta a punto de técnicas de reforzamiento que permitan abordar descensos poblacionales, o de reintroducción para hacer frente a una hipotética desaparición de un núcleo poblacional. Ensayos piloto desarrollados por nuestro equipo han logrado establecer una pequeña población de $C$. rupestris subsp. rupestris en la Sierra de Alcaraz (provincia de Albacete). Para ello los resultados obtenidos en el presente trabajo (principalmente los relativos a tiempo de desarrollo de los taxones en cultivo e inicio de la floración) han sido de utilidad al permitirnos diseñar una programación temporal de las actuaciones en vivero y posteriormente en campo. El trasplante de los ejemplares conviene realizarlo lo más tarde posible para reducir la probabilidad de daños por herbivoría, pero con anterioridad al inicio de la floración para conceder a la planta un pequeño periodo de adaptación que le permita una captación de recursos lo más eficiente posible al comienzo de esta. Así se posibilita un aceptable éxito reproductivo que puede suponer la persistencia del taxón en el área exclusivamente por medio de reclutamiento natural.

\section{AGRADECIMIENTOS}

Este trabajo ha sido financiado por la Junta de Comunidades de Castilla-La Mancha a través de los proyectos de referencias PREG-00-097 y PAI07-00880300, y por el Ministerio de Ciencia e Innovación del Gobierno de España mediante el proyecto de referencia CGL2009-08723. Asimismo, queremos expresar nuestro más sincero agradecimiento al profesor César Gómez-Campo por los consejos dados a la hora de abordar este trabajo.

\section{LITERATURA CITADA}

BAKER-BROSH KF \& RK PEET (1997) The ecological significance of lobed and toothed leaves in temperate forest trees. Ecology 78: 1250-1255.

BASKIN JM \& CC BASKIN (1988) Endemism in rock outcrop plant communities of unglaciated eastern United States: An evaluation of the roles of the edaphic, genetic and light factors. Journal of Biogeography 15: 829-840.

BASKIN JM, KM SNYDER, JL WALCK \& CC BASKIN (1997) The comparative autecology of endemic, globally-rare and geographically-widespread, common plant species: Three case studies. Southwestern Naturalist 42: 384-399.

BOSSDORF O, S SCHRÖDER, D PRATI \& H AUGE (2004) Palatability and tolerance to simulated herbivory in native and introduced populations of Alliaria petiolata (Brassicaceae). American Journal of Botany 91: 856-862.

CASTROVIEJO S, M LAÍNZ, G LÓPEZ-GONZÁLEZ, P MONTSERRAT, F MUÑOZ-GARMENDIA et al. (eds) (1986-2003) Flora Iberica. Plantas vasculares de la Península Ibérica e Islas Baleares. Issues I-XIV. Real Jardín Botánico, CSIC, Madrid.

CHOUARD P (1960) Vernalization and its relations to dormancy. Annual Review of Plant Physiology 11: 191-238.

COPETE MA (2005) Aspectos de la biología poblacional y reproductiva de las especies vegetales amenazadas Helianthemum polygonoides, Coincya rupestris subsp. rupestris, Sideritis serrata y Sisymbrium cavanillesianum. Tesis Doctoral. Escuela Técnica Superior de Ingenieros Agrónomos de Albacete, Universidad de Castilla-La Mancha.

COPETE MA, JM HERRANZ \& P FERRANDIS (2005) Seed dormancy and germination in threatened Iberian Coincya (Brassicaceae) taxa. Ecoscience 12: 257-266.

COPETE MA, JM HERRANZ \& P FERRANDIS (2008) Reproductive biology of the critically endangered endemic Mediterranean plant Coincya rupestris subsp. rupestris (Spain): The effects of competition and summer drought on seedling establishment. Revista Chilena de Historia Natural 81: 345-359.

DATA TRANSLATION INC (2001) Global Lab Image/2 version 3.0. Marlboro, USA.

DOCE (1992) Directive 92/43/CEE concerning the conservation of habitats and wild fauna and flora. DOCE/L 206, 22-04-92. Council of the European Communities, Brussels, Belgium.

DOCM (1999) Decreto 237/1999 de 14 de diciembre, por el que se aprueba el Plan de Recuperación 
de la especie de flora Coincya rupestris subsp. rupestris. DOCM de 30 de diciembre de 1999. Consejería de Administraciones Públicas y Justicia, Toledo, España.

ERWIN J (2005) Factor affecting flowering in ornamental plants. En: McDonald MB \& FY Kwong (eds) Flower seeds. Biology and technology: 87-116. CAB International, Wallingford.

GIVNISH TJ (1987) Comparative studies of leaf form assessing the relative roles of selective pressures and phylogenetic constraints. New Phytologist 106: 131-160.

GÓMEZ-CAMPO C (1977) Clinal variation and evolution in the Hutera-Rhynchosinapis complex of the Sierra Morena (south-central Spain). Botanical Journal of the Linnean Society 75: 179-194.

GÓMEZ-CAMPO C, JM HERRANZ \& F MONTERO (2001) The genus Coincya Rouy (Cruciferae) in south-central Spain revisited: A morphometric analysis of population structure. Botanical Journal of the Linnean Society 135: 125-135.

HERRANZ JM, P FERRANDIS \& MA COPETE (2003) Influence of temperature, maternal source, and seed position in fruit on seed germination and ability to form soil seed banks in threatened species of Coincya (Cruciferae). Israel Journal of Plant Sciences 51: 133-141.

HERRANZ JM, P FERRANDIS \& JJ MARTÍNEZSÁNCHEZ (1996) Propuesta del plan de recuperación de Coincya rupestris Porta \& Rigo ex Rouy subsp. rupestris. Inéd. Junta de Comunidades de Castilla-La Mancha.

HICKEY LJ \& JA WOLFE (1975) The bases of angiosperm phylogeny: Vegetative morphology. Annals of the Missouri Botanical Garden 62: 538589.

KASA GR \& ZP KONDRA (1986) Growth analysis of springtype oilseed rape. Field Crops Research 14: 361-370.

KINCAID DT \& RM SCHNEIDER (1983) Quantification of leaf shape with a microcomputer and Fourier transform. Canadian Journal of Botany 61: 2333-2342.

KRANNITZ PG, LW AARSSEN \& JF DOW (1991) The effect of genetically based differences in seed size on seedling survival in Arabidopsis thaliana (Brassicaceae). American Journal of Botany 78: 446-450.

LAVERGNE S, E GARNIER \& M DEBUSSCHE (2003) Do rock endemic and widespread plant species differ under the Leaf-Height-Seed plant ecology strategy scheme? Ecology Letters 6: 398-404.

LEADLAY EA (1993) Coincya Rouy. En: Castroviejo S, C Aedo, C Gómez-Campo, $\mathrm{M}$ Laínz, $\mathrm{P}$ Montserrat et al. (eds) Flora Iberica. Plantas vasculares de la Península Ibérica e Islas Baleares Vol. IV: 400-411. Real Jardín Botánico, CSIC, Madrid.

LÜSCHER A, B STÄHELI, R BEAUN \& J NÖSBERGER (2001) Leaf area, competition with grass, and clover cultivar: Key factors to successful overwintering and fast regrowth of white clover (Trifolium repens L.) in spring. Annals of Botany 88: $725-735$
MAUN MA \& AM PAYNE (1998) Fruit and seed polymorphism and its relation to seedling growth in the genus Cakile. Canadian Journal of Botany 76: 2743-2750.

MARAÑÓN T \& PJ GRUBB (1993) Physiological basis and ecological significance of the seed size and relative growth rate relationship in Mediterranean annuals. Functional Ecology 7: 591-599.

McLELLAN T (1993) The roles of heterochrony and heteroblasty in the diversification of leaf shapes in Begonia dregei (Begoniaceae). American Journal of Botany 80: 796-804.

McLELLAN T (2000) Geographic variation and plasticity of leaf shape and size in Begonia dregei and B. homonyma (Begoniaceae). Botanical Journal of the Linnean Society 132: 79-95.

McLELLAN T (2005) Correlated evolution of leaf shape and trichomes in Begonia dregei (Begoniaceae). American Journal of Botany 92: 1616-1623.

McLELLAN T \& JA ENDLER (1998) The relative success of some methods for measuring and describing the shape of complex objects. Systematic Biology 47: 264-281.

MORENO JC (coordinador) (2008) Lista Roja 2008 de la flora vascular española. Dirección General de Medio Natural y Política Forestal. Ministerio de Medio Ambiente, y Medio Rural y Marino, y Sociedad Española de Biología de la Conservación de Plantas, Madrid.

NANDA R, SC BHARGAVA \& HM RAWSON (1995) Effect of sowing date on rates of leaf appearance, final leaf numbers and areas in Brassica campestris, B. juncea, B. napus and B. carinata. Field Crops Research 42: 125-134

TSUDA M (1999) Errors in leaf area measurement with an automatic area meter due to leaf chlorophyll in crop plants. Annals of Botany 84: 799-801.

TUKEY JW (1949) Comparing individual means in the analysis of variance. Biometrics 5: 99-114.

UICN (2001) Categorías y criterios de la lista roja de la UICN: versión 3.1. Comisión de Supervivencia de Especies de la UICN, Gland, Suiza y Cambridge, Reino Unido.

WALCK JL, JM BASKIN \& CC BASKIN (1999) Relative competitive abilities and growth characteristics of a narrowly endemic and a geographically widespread Solidago species (Asteraceae). American Journal of Botany 86: 820-828.

WALCK JL, JM BASKIN \& CC BASKIN (2001) Why is Solidago shortii narrowly endemic and $S$. altissima geographically widespread? A comprehensive comparative study of biological traits. Journal of Biogeography 28: 1221-1237.

WALTER H (1977) Zonas de vegetación y clima. Editorial Omega, Barcelona.

WILF P (1997) When are leaves good thermometers? A new case for leaf margin analysis. Paleobiology 23: 373-390.

WITKOWSKI ETF \& BB LAMONT (1997) Does the rare Banksia goodii have inferior vegetative, reproductive or ecological attributes compared with its widespread co-ocurring relative $B$. gardneri? Journal of Biogeography 24: 469-482. 